\title{
When Primary Care Providers (PCPs) Help Patients Choose Prostate Cancer Treatment
}

\author{
Archana Radhakrishnan, MD, MHS, David Grande, MD, MPA, Michelle Ross, PhD, \\ Nandita Mitra, PhD, Justin Bekelman, MD, Christian Stillson, MPH, \\ and Craig Evan Pollack, MD, MHS
}

Background: The role of primary care providers (PCPs) in decision making around cancer care remains largely unknown. We evaluated how frequently men with localized prostate cancer report receiving help from their PCP about their treatment, and whether those men who do are less likely to receive definitive treatment.

Methods: We mailed surveys to men newly diagnosed with localized prostate cancer between 2012 and 2014 in the greater Philadelphia region. Participants were asked whether their PCP helped decide how to treat their cancer. The outcome was receipt of definitive treatment (either radical prostatectomy or radiotherapy).

Results: A total of 2386 men responded (adjusted response rate, 51.1\%). Among these men, 38.2\% reported receiving help from their PCP regarding choosing a treatment, and $79.6 \%$ received definitive treatment. In adjusted analyses, non-Hispanic black men (odds ratio, 1.76; 95\% confidence interval, 1.37-2.27) were more likely than non-Hispanic white men to report receiving help from their PCP. However, men who did receive help were not more likely to forgo definitive treatment overall $(P=.58)$ or in the subgroups of men who may be least likely to benefit from definitive treatment.

Conclusions: Though a substantial proportion of men reported receiving help from their PCP about prostate cancer treatment, these discussions were not associated with different treatment patterns. Further effort is needed to determine how to optimize the role of PCPs in supporting patients to make preference-sensitive cancer decisions. (J Am Board Fam Med 2017;30:298-307.)

Keywords: Philadelphia, Primary Care Physicians, Primary Health Care, Prostate Cancer, Surveys and Questionnaires

In 2013, the Institute of Medicine called for collaboration between cancer specialists and primary care providers (PCPs) to improve delivery of com-

This article was externally peer reviewed.

Submitted 11 November 2016; revised 30 January 2017; accepted 6 February 2017.

From the Division of General Internal Medicine, Johns Hopkins University, Baltimore, MD (AR, CEP); the Division of General Internal Medicine, Hospital of the University of Pennsylvania Philadelphia (DG, CS); the Department of Biostatistics and Epidemiology, Hospital of the University of Pennsylvania (MR, NM); the Department of Radiation Oncology, Hospital of the University of Pennsylvania (JB); and the Department of Epidemiology, Johns Hopkins Bloomberg School of Public Health, Baltimore (CEP).

Funding: This work was supported by the National Institute on Minority Health and Health Disparities (P60 MD006900). AR's salary is supported by the National Heart, Lung, and Blood Institute (T32H1007180). CEP's salary is supported by the National Cancer Institute (K07CA151910). JB's salary is supported by the National Cancer Institute (K07CA163616). prehensive, high-quality cancer care. ${ }^{1}$ This was motivated, in part, by recognizing the growing number of cancer patients with complex medical needs. The role of PCPs in cancer care has typically been limited to screening, diagnosis, and, more recently, survivorship., ${ }^{2,3}$

Studies evaluating PCP involvement in cancer treatment decision making remain limited. ${ }^{4,5}$ Wallner and colleagues ${ }^{5}$ recently found that $35 \%$ of women with breast cancer reported a high level of PCP participation in their treatment decision, but this was not with linked with more meaningful

Conflict of interest: none declared.

Corresponding author: Archana Radhakrishnan, MD, MHS, Division of General Internal Medicine, Johns Hopkins University, 2024 E. Monument St, Suite 2-300C, Baltimore, MD 21287 (E-mail: aradhak3@jhu.edu). 
deliberation for their treatment decision. Jang and colleagues $^{4}$ used cancer registry and Medicare claims data to evaluate how PCP visits relate to treatment choice, finding that $22 \%$ of men visited their PCP before their prostate cancer treatment and were more likely to receive conservative management. However, that study used any visit with a PCP before treatment, as identified in insurance claims, as a proxy for treatment discussions and did not evaluate the effect among subgroups of men eligible for active surveillance.

Building on the prior literature and drawing on a large survey of men diagnosed with localized prostate cancer, we sought to better understand how frequently men reported receiving help from their PCP in deciding on prostate cancer treatment and whether reporting help was linked with different patterns of treatment. Men with clinically localized prostate cancer represent an important clinical group in which to evaluate the potential role of PCPs in treatment decisions. Prostate cancer is diagnosed in 180,890 men in the United States annually, ${ }^{6}$ and men with localized disease face a choice among treatment options-including radical prostatectomy, radiation therapy, and active surveillance-with varying side effects and benefits. Ideally, patients' preferences, in conjunction with clinical features, should guide the treatment that they receive. ${ }^{7,8}$ In practice, however, treatment often depends on the type of specialist that a patient sees. Hoffman and colleagues ${ }^{9}$ found that the urologist who diagnosed a patient's prostate cancer accounted for twice as much of the variation in his ultimate treatment when compared with patientlevel characteristics. When cancer specialists are surveyed regarding their management of prostate cancer, specialists tend to recommend the treatment that they themselves deliver: the majority of urologists recommend radical prostatectomy for clinically localized cancer, whereas the majority of radiation oncologists recommend radiation therapy. $^{10-12}$

PCPs may be in an ideal position to help men with prostate cancer make treatment decisions. First, compared with specialists, PCPs are more likely to have longitudinal relationships with their patients and often act as trusted sources of information and provide emotional support for their patients. ${ }^{13-15}$ In this role, they may be better suited to elicit patient preferences and consider patient comorbidities in clinical decision making. ${ }^{16-19}$ Sec- ond, unlike specialists who are apt to recommend the treatment they themselves deliver, PCPs may be more likely to deliver balanced treatment recommendations and thereby help patients make treatment decisions consistent with individual values. ${ }^{2,20}$ A PCP's role may be particularly important for patients who qualify for active surveillance programs based on their age, comorbidities, functional status, and stage of their prostate cancer.

In this study, we hypothesized that men with localized prostate cancer who received help from their PCP to choose a treatment would be less likely to receive definitive treatment with either radical prostatectomy or radiation therapy, ${ }^{4}$ and we anticipated that the effect would be larger among men who may benefit most from active surveillance, particularly men who are of advanced age, who have a shorter life expectancy, and who have lower-risk tumors. ${ }^{21}$ In addition, with well-documented racial differences in physician-patient communication and patients' engagement in their own health care, ${ }^{22,23}$ we hypothesized that black men would be less likely to report receiving help from their PCP before treatment, and that these conversations may be less strongly associated with the type of treatment they receive.

\section{Methods \\ Patient Cobort}

The Philadelphia Area Prostate Cancer Access Study ( $\mathrm{P}^{2}$ Access) is a study of black and white men diagnosed with localized prostate cancer within the greater Philadelphia area. This study uses multiple data sources, including a state cancer registry, patient surveys, and provider inventories, to understand how access to care influences racial differences in prostate cancer treatment. This article is a secondary analysis of data from the patient survey. Men diagnosed with prostate cancer between January 1, 2012, and December 31, 2014, were identified from the Pennsylvania Cancer Registry (PCR) using the following inclusion criteria: (1) new diagnosis of prostate cancer; (2) adenocarcinoma histology; and (3) resident of 8 specified counties within the greater Philadelphia area (Berks, Bucks, Chester, Delaware, Lancaster, Lehigh, Montgomery, and Philadelphia). Men were excluded if they (1) died before data could be collected, (2) were unable to speak English or Spanish, and (3) had metastatic disease at the time of pre- 
sentation. We also excluded men if they had military insurance (including Tricare and Veterans Administration) and received chemotherapy for treatment. This article focuses specifically on the subset of men who reported having a PCP at the time of their cancer diagnosis $(95 \%$ of the total respondents).

\section{Patient Questionnaire}

Men who were newly diagnosed with prostate cancer were invited to participate in a survey between February 2014 and September 2015, reflecting the lag time from diagnosis to identifying patients and obtaining data from the PCR. Participants received up to 2 mailings of the survey, followed by phone calls to all nonresponders. An unconditional \$2 incentive was included in the initial mailing, and $\$ 15$ was provided upon completion of the survey. Survey items were based on prior studies ${ }^{4,24-27}$ and were pilot tested among a similar cohort of men with newly diagnosed localized prostate cancer.

\section{PCP Help with Treatment Decision Making}

To assess the role of PCPs in prostate cancer treatment decision making, men were asked in the survey, "After you found out you had prostate cancer, did your primary care provider help you decide how to treat it?" Responses were yes or no.

\section{Treatment}

Data from the PCR was used to define receipt of definitive treatment. Definitive treatment was classified as having either radical prostatectomy or radiotherapy (including external beam radiation therapy or seed brachytherapy).

\section{Covariates}

Patient sociodemographic characteristics that have previously been found to influence prostate cancer treatment were obtained from the survey and the cancer registry. From the survey, patients reported their education (less than eighth grade, some high school, high school graduate, some college, college graduate, and beyond college education), race/ethnicity (non-Hispanic white, non-Hispanic black, Hispanic, and non-Hispanic other), and marital status (married or single/other). ${ }^{10,28}$ We obtained insurance at the time of diagnosis from the PCR, classified as private, Medicaid, Medicare (with and without a supplement), and none.
A validated mortality index was used to calculate the life expectancy for each respondent based on survey data. ${ }^{25}$ The index included age, body mass index, tobacco use, self-reported comorbidities, and functional status. Participants were grouped into those with low $(<25 \%)$, intermediate $(25-$ $50 \%)$, high $(50-75 \%)$, and very high $(\geq 75 \%)$ risks of 10 -year mortality. ${ }^{29}$

Tumor-specific data were abstracted from the cancer registry. For our primary analysis, we evaluated a Gleason score from biopsy $(<7,7,>7)$, and the clinical tumor stage (stage 1, 2, or 3 based on the American Joint Committee on Cancer TNM system). ${ }^{30}$ We performed a sensitivity analysis calculating risk based on National Comprehensive Cancer Network classification (NCCN) (low, intermediate, and high risk), which further included prostate-specific antigen results obtained from the PCR. Gleason score, clinical tumor stage, and NCCN risk stratification have all been previously shown to be prognostic predictors of prostate cancer outcomes. $^{21,31}$

\section{Statistical Analysis}

Descriptive analysis was used to provide summaries of patient sociodemographic and tumor characteristics. The $\chi^{2}$ test was used to compare characteristics of men who did and did not receive help from their PCP to decide on prostate cancer treatment following diagnosis. We constructed a multivariable logistic regression model to assess whether receiving help from the PCP regarding treatment was associated with patient sociodemographic (age, race/ethnicity, education, insurance, employment, and marital status) and clinical (life expectancy, Gleason score, and clinical tumor stage) characteristics. Next, we examined the association between receiving help from the PCP and receipt of definitive treatment. Propensity score matching was used to achieve balance on observable characteristics among those who did and did not receive help from their PCP. This was implemented using 1-to-1 nearest-neighbor matching and doubly robust methods in our multivariable regression model. ${ }^{32}$ Our initial model adjusted for the abovementioned sociodemographic and clinical characteristics. We then assessed whether the relationship between receiving help from the PCP and treatment patterns varied by patient race by including an interaction term (race $\times$ PCP involvement). We performed 3 sets of subgroup analyses to examine 
Table 1. Demographic and Clinical Characteristics of Patients

Characteristic

Age (years)

Mean (SD)

$<60$

$60-64$

65-69

$70-74$

$\geq 75$

Missing

Race/ethnicity

Non-Hispanic white

Non-Hispanic black

Hispanic

Non-Hispanic other

Missing

Education

Less than high school

High school graduate

Some college

College graduate

More than college

Missing

Insurance

Private

Medicaid

Medicare

None

Missing

Employment

Unemployed/other

Employed

Retired

Missing

Marital status

Single

Married

Missing

$10-Y$ ear risk of mortality

$<25 \%$

$25-50 \%$

50-75\%

$\geq 75 \%$

Missing

Gleason score

$<7$

7

$>7$

Missing
$65.4(8.3)$

567 (24.7)

$459(20.0)$

$570(24.9)$

$363(15.8)$

318 (13.9)

$17(0.7)$

1804 (78.6)

$351(15.3)$

41 (1.8)

$26(1.1)$

$72(3.1)$

157 (6.8)

$529(23.1)$

463 (20.2)

$349(15.2)$

$620(27.0)$

$176(7.7)$

$1271(55.4)$

67 (2.9)

$921(40.1)$

$10(0.4)$

25 (1.1)

242 (10.6)

$902(39.3)$

$972(42.4)$

$178(7.8)$

425 (18.5)

$1836(80.0)$

33 (1.4)

$622(27.1)$

747 (32.6)

$548(23.9)$

$225(9.8)$

$152(6.6)$

$6.8(0.89)$

$909(39.6)$

$920(40.1)$

$400(17.4)$

$65(2.8)$

Continued
Table 1. Continued

\begin{tabular}{lc}
\hline Characteristic & \\
\hline Clinical tumor stage & \\
1 & $1642(71.6)$ \\
2 & $558(24.3)$ \\
3 & $51(2.2)$ \\
Missing & $43(1.9)$ \\
Definitive treatment & \\
Yes & $1825(79.6)$ \\
No & $364(15.8)$ \\
Missing & $105(4.6)$ \\
Treatment & \\
Surgery & $1175(51.2)$ \\
Radiation therapy & $680(29.6)$ \\
Hormone therapy & $309(13.5)$ \\
\hline
\end{tabular}

Data are $\mathrm{n}(\%)$ of patients unless otherwise indicated. $\mathrm{SD}$, standard deviation.

the association between receiving help from the PCP and receipt of definitive treatment among men who would be considered suitable candidates for active surveillance under clinical guidelines: (1) age $>70$ years; (2) limited life expectancy (10-year risk of mortality $>50 \%$ ); and (3) NCCN-classified low-risk prostate cancer. ${ }^{21,33}$ Finally, among the subgroup of men who received definitive treatment, we examined whether receiving help from the PCP was associated with either type of treatment (surgery or radiation) using a multivariable logistic regression model, adjusting for sociodemographic and clinical characteristics. For covariates with missing data, we used multiple imputation via multiple chained equations based on all available patient characteristics. Five imputed data sets were used in this approach. Analyses were conducted using Stata 13.0 software (StataCorp, College Station, TX). This study was approved by the institutional review boards at the University of Pennsylvania and Johns Hopkins University.

\section{Results}

A total of 2386 of 4672 eligible men completed the survey (adjusted response rate, 51.1\%). Compared with nonresponders, responders were white $(77.5 \%$ vs $61.5 \%$ ) and had higher rates of receiving definitive treatment $(79.5 \%$ vs $70.9 \%$ ) (Appendix Table 1). Among the 2294 men in our final analytic cohort, the average age was 65.4 years (standard deviation, 8.3 years; Table 1). Men predominantly were white $(78.6 \%)$, were married $(80.0 \%)$, had a 
higher-level education (62.4\%), and had either private insurance $(55.4 \%)$ or Medicare (40.1\%). The majority of men had tumors with a Gleason score $\leq 7$ and clinical tumor stage 1 disease.

Over a third of men $(38.2 \%)$ reported receiving help from their primary care provider in deciding how to treat their prostate cancer (Table 2). In bivariate analyses, black men were more likely to have reported receiving help compared with white men. PCP involvement was less likely among men who were employed or retired compared with those who were unemployed. In the adjusted analysis, black men (odds ratio, 1.76; 95\% confidence interval, 1.37-2.27) had a significantly higher likelihood of receiving help from their PCP compared with white men (Table 2).

Overall, 1825 men (79.6\%) received definitive treatment; $38.8 \%$ of men who received definitive treatment reported receiving help from their PCP, compared with $40.3 \%$ of men who did not receive treatment $(P=.58)$. Using propensity score matching, we achieved appropriate balance on observable covariates. In our doubly robust models, men who received help from their PCP were as likely to undergo definitive treatment as men who did not report receiving help (odds ratio, $0.97,95 \%$ confidence interval, 0.75-1.26). Race did not modify the effect of PCP involvement on definitive treatment $(P=.79)$. In subgroup analyses of men more likely to be eligible for active surveillance-including men with NCCN-classified low-risk disease, with limited life expectancy ( $\geq 50 \% 10$-year risk of mortality), and $>70$ years old-we did not find evidence that discussions with PCPs were associated with receipt of definitive treatment (Figure 1). Further, we did not find significant associations between PCP help and the specific type of treatment (surgery or radiation) among men who received definitive treatment.

\section{Discussion}

In a large, population-based sample of men with localized prostate cancer, we found that over one third reported receiving help from their PCP in deciding how to treat their cancer. In particular, black men were more likely to report receiving help from their PCP compared with white men. Contrary to our original hypothesis, we did not find evidence that men who reported receiving help from their PCP were less likely to receive definitive therapy, either overall or in subgroups of men most likely to be considered for active surveillance based on clinical guidelines. Past studies show that PCP involvement is often limited during cancer treatment. ${ }^{15,34,35}$ Our results may suggest opportunities to increase the role of PCPs in treatment decision making among men with localized prostate cancer.

In our sample, a larger proportion of men (38\%) reported receiving help than was found by Jang and colleagues $^{4}(22 \%)$. Some of the difference may stem from differences in approaches to measuring PCP involvement. Jang et al used visit patterns from the Surveillance, Epidemiology and End Results/Medicare database and defined PCP involvement as a visit to a PCP between diagnosis and treatment. We rely on patient self-report of receiving help during office visits or through other modes such as telephone or electronic communication. Further, in contrast to the study by Jang et al, we limited our cohort to men who reported having a PCP at the time of their prostate cancer diagnosis. It is notable that, in our study, black men were more likely to report discussing treatment with their PCP, despite evidence suggesting that these men are less likely to be active participants in their health care and to be engaged with their providers. ${ }^{23,36}$ Our results may suggest that PCP involvement is greater for patients vulnerable to disparities in care. They may also point to potential differences between black and white patients in what they value for high-quality care; similar to findings from prior studies, minority patients may value their PCP's holistic approach to cancer care rather than disease-specific care. ${ }^{37,38}$ Future efforts to empower PCPs to be able to engage with patients in cancer treatment discussions is critical.

We did not find that receiving help from PCPs was associated with a lower likelihood of receiving definitive treatment. Our results were in contrast to those of Jang and colleagues, ${ }^{4}$ though in that study it is uncertain whether patients discussed their options for prostate cancer treatment during the PCP visit. It may be that visit patterns with PCPs around the time of diagnosis and treatment are related to other factors that might influence the decision of whether to pursue definitive treatment. Patients perceived their primary care to be of high quality in the study by Wallner et al, ${ }^{5}$ yet PCP engagement, communication, and participation were not associated with improvement in meaningful deliberation over treatment decisions. Within this context, lack 
Table 2. Bivariate and Multivariable Analyses of Patient Characteristics Associated with Receiving Help from the Primary Care Physician Regarding Treatment

\begin{tabular}{|c|c|c|c|c|c|}
\hline & \multicolumn{2}{|c|}{$\begin{array}{l}\text { Received Help from the PCP, } \\
\mathrm{n}(\%)\end{array}$} & \multirow[b]{2}{*}{$P$ Value* } & \multirow{2}{*}{$\begin{array}{c}\text { Adjusted Model, }{ }^{\dagger} \text { Odds Ratio } \\
(95 \% \mathrm{CI})\end{array}$} & \multirow[b]{2}{*}{$P$ Valuc } \\
\hline & No & Yes & & & \\
\hline Total & $1375(59.9)$ & $876(38.2)$ & & & \\
\hline Age (years) & & & .24 & & \\
\hline$<60$ & $340(24.8)$ & $220(25.3)$ & & Reference & \\
\hline $60-64$ & $276(20.1)$ & $175(20.1)$ & & $0.96(0.72-1.27)$ & .77 \\
\hline $65-69$ & $363(26.4)$ & $200(23.0)$ & & $0.75(0.51-1.09)$ & .13 \\
\hline $70-74$ & $220(16.0)$ & $141(16.2)$ & & $0.78(0.51-1.19)$ & .25 \\
\hline$\geq 75$ & $175(12.7)$ & $135(15.5)$ & & $0.85(0.55-1.35)$ & .50 \\
\hline Race/ethnicity & & & $<.001$ & & \\
\hline Non-Hispanic white & $1144(85.1)$ & $637(75.3)$ & & Reference & \\
\hline Non-Hispanic black & $167(12.4)$ & $177(20.9)$ & & $1.76(1.37-2.27)$ & $<.001$ \\
\hline Hispanic & $20(1.5)$ & $20(2.4)$ & & $1.72(0.91-3.28)$ & .10 \\
\hline Non-Hispanic other & $13(1.0)$ & $12(1.4)$ & & $1.48(0.68-3.22)$ & .33 \\
\hline Education & & & .04 & & \\
\hline $\begin{array}{l}\text { Less than high } \\
\text { school }\end{array}$ & $75(5.9)$ & $73(9.1)$ & & Reference & \\
\hline $\begin{array}{l}\text { High school } \\
\text { graduate }\end{array}$ & $316(24.8)$ & $205(25.6)$ & & $0.86(0.61-1.26)$ & .48 \\
\hline Some college & $275(21.6)$ & $179(22.3)$ & & $0.92(0.63-1.36)$ & .69 \\
\hline College graduate & $214(16.8)$ & $127(15.8)$ & & $0.97(0.64-1.46)$ & .87 \\
\hline More than college & $396(31.0)$ & $218(27.2)$ & & $0.91(0.62-1.32)$ & .62 \\
\hline Insurance & & & .28 & & \\
\hline Private & $783(57.5)$ & $469(54.3)$ & & Reference & \\
\hline Medicaid & $34(2.5)$ & $31(3.6)$ & & $0.87(0.50-1.50)$ & .61 \\
\hline Medicare & $539(39.6)$ & $361(41.8)$ & & $1.04(0.82-1.33)$ & .74 \\
\hline None & $6(0.4)$ & $3(0.4)$ & & $0.66(0.16-2.70)$ & .56 \\
\hline Employment & & & $<.001$ & & \\
\hline Unemployed/other & $117(9.2)$ & $116(14.5)$ & & Reference & \\
\hline Employed & $579(45.3)$ & $309(38.6)$ & & $0.75(0.54-1.04)$ & .09 \\
\hline Retired & $581(45.5)$ & $376(46.9)$ & & $0.83(0.59-1.14)$ & .24 \\
\hline Marital Status & & & .08 & & \\
\hline Single & $241(17.7)$ & $179(20.7)$ & & Reference & \\
\hline Married & $1122(82.3)$ & $688(79.4)$ & & $1.03(0.81-1.30)$ & .84 \\
\hline 10 -Year mortality risk & & & $<.001$ & & \\
\hline$<25 \%$ & $395(30.3)$ & $221(27.3)$ & & Reference & \\
\hline $25-50 \%$ & $486(37.2)$ & $250(30.9)$ & & $1.10(0.79-1.54)$ & .54 \\
\hline $50-75 \%$ & $299(22.9)$ & $244(30.1)$ & & $1.62(1.11-2.36)$ & .01 \\
\hline$\geq 75 \%$ & $125(9.6)$ & $95(11.7)$ & & $1.36(0.87-2.13)$ & .18 \\
\hline Gleason score & & & .73 & & \\
\hline$\leq 6$ & $554(41.3)$ & $336(39.6)$ & & Reference & \\
\hline 7 & $549(40.9)$ & $359(42.3)$ & & $1.03(0.84-1.25)$ & .77 \\
\hline$>7$ & $238(17.8)$ & $153(18.0)$ & & $1.03(0.80-1.34)$ & .80 \\
\hline Clinical tumor stage & & & .92 & & \\
\hline 1 & $984(72.8)$ & $627(73.0)$ & & Reference & \\
\hline 2 & $335(24.8)$ & $214(24.9)$ & & $0.99(0.80-1.22)$ & .93 \\
\hline 3 & $32(2.4)$ & $18(2.1)$ & & $0.88(0.48-1.62)$ & .68 \\
\hline
\end{tabular}

${ }^{*} \chi^{2}$ Test.

${ }^{\dagger}$ Imputed data, adjusted for age, race, education, insurance, employment, marital status, life expectancy, Gleason score, and clinical stage.

CI, confidence interval. 
Figure 1. Adjusted odds ratio (OR) and $95 \%$ confidence interval of receipt of definitive treatment associated with receiving help from the primary care physician among various patient cohorts. All models adjusted for age, race, education, insurance, employment, marital status, and life expectancy, with models for older men and limited life expectancy additionally adjusted for Gleason score and clinical stage. $\mathrm{NCCN}=$ National comprehensive Cancer Network.

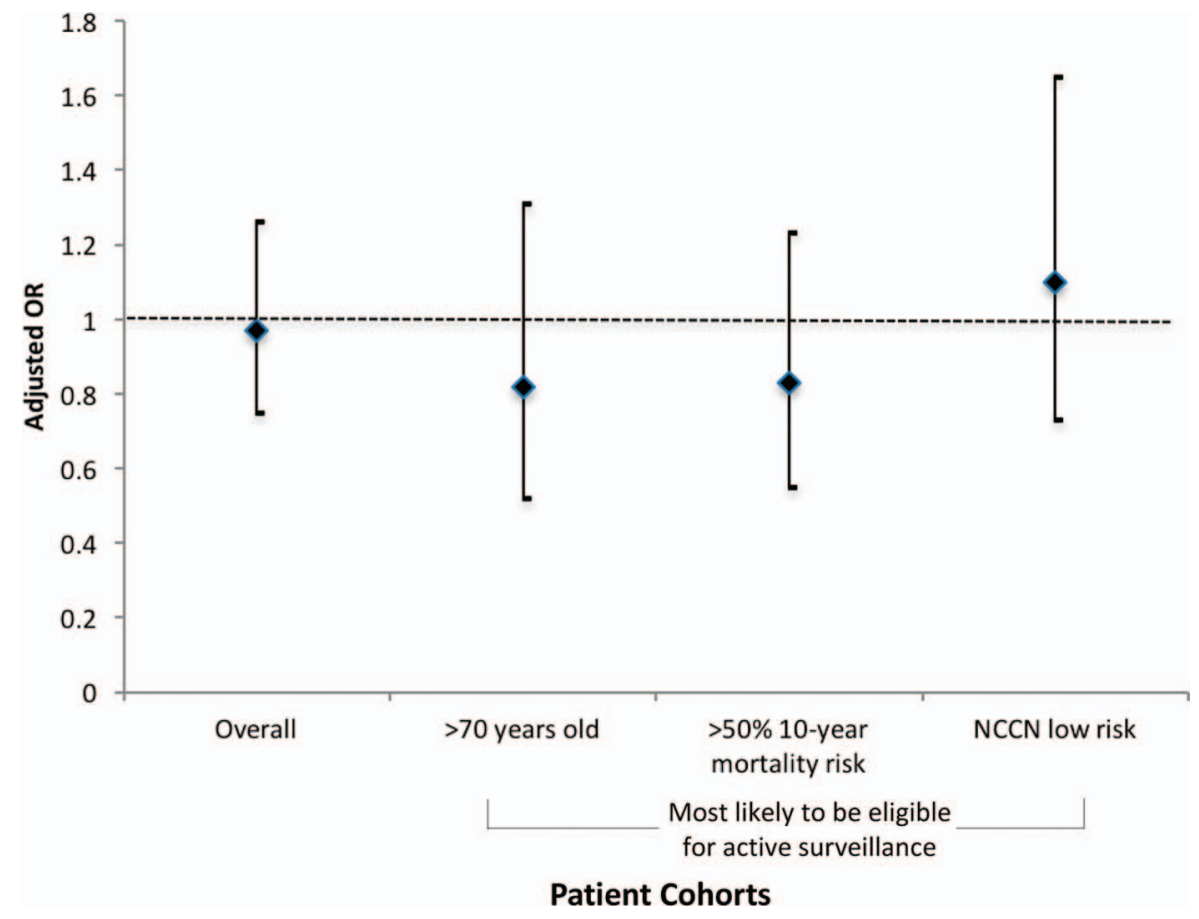

of association with differences in treatment patterns as a result of PCP involvement may reflect the limited influence of PCPs during treatment decision making, a period in which cancer specialists' recommendations carry the most weight with patients. In our study, we also did not find PCP involvement in decision making to be associated with different treatment patterns among men who may be least likely to benefit from definitive treatment, including older men, men with limited life expectancy, and men with low-risk disease. Many of these men receive definitive treatment and are often unaware of the risk-benefit controversies associated with treatments. ${ }^{39-41}$ PCPs may be particularly well suited to capitalize on their longitudinal relationship with patients within these subgroups of men, using their knowledge of the patient's comorbidities and functional status to help ensure that the patient is making decisions in line with their values and preferences.

With a large portion of patients reporting receiving help but no evidence from our data that, at a population-level, this affected treatment patterns, further work is needed to determine whether and how PCPs' roles might be extended to have a greater influence in the treatment decision-making process. In a survey of PCPs and cancer specialists, Klabunde and colleagues ${ }^{34}$ found that many PCPs saw themselves as playing a role in providing general medical care, but far fewer reported a role in determining initial cancer treatment. PCPs also report feeling disconnected from their patient's care and endorse having insufficient knowledge about treatment plans. ${ }^{14,42,43}$ This may limit a PCP's ability to effectively help his or her patients navigate complex cancer treatment decisions. New models of care that integrate interdisciplinary cancer care teams, promote communication between PCPs and cancer specialists, and improve provider competencies around cancer treatment may empower PCPs to engage in more meaningful discussions with their patients about key treatment decisions.

This study has several limitations. First, while men reported obtaining help from their PCP in choosing a treatment, we are unable to determine the ways in which PCPs helped. It is possible that 
some PCPs engaged in discussing the risks and benefits of different treatment options and assessing patient preferences, others may have provided emotional support, and still others may have had more limited conversations. Delving deeper into the type of help provided-both from a patient and a PCP perspective-and analyzing the content of conversations around these issues is an important next step. Second, we do not have information on the duration of the PCP-patient relationship, which may affect the quality of the discussions and the influence of the PCP on treatment decisions. We might expect that longer relationships are associated with greater provider trust and more influence on decision making. Third, nonresponse bias may have affected our findings, as responders were more likely to be white men and received definitive treatment. With conflicting prior evidence, ${ }^{4,5}$ the direction in which this would bias our findings on the association between PCP involvement and definitive treatment remains unclear. Fourth, patient self-report of discussions with their PCP could be susceptible to recall and social desirability biases; however, they are similar to those reported in prior studies. ${ }^{5,20}$ Fifth, data were obtained from a single geographic area, which may limit the generalizability of the study. However, the study area includes 5.3 million residents across urban and suburban locales. It is racially and ethnically diverse, and $29 \%$ of the area's population is nonwhite.

\section{Conclusions}

Receiving help from a PCP was not associated with differences in treatment patterns in our study population of men with clinically localized prostate cancer, either overall or in cohorts who may be least likely to benefit from definitive treatment. Our study may point to opportunities to leverage the help that PCPs are providing to their patients in order to promote preference-sensitive care, and also the need to explore new models of collaborative decision making between cancer specialists, PCPs, and patients.

To see this article online, please go to: http://jabfm.org/content/ 30/3/298.full.

\section{References}

1. Institute of Medicine; Board on Health Care Services; Committee on Improving the Quality of Cancer Care: Addressing the Challenges of an Aging
Population; Levit LA, Balogh EP, Nass SJ, Ganz PA, eds. Delivering high-quality cancer care: charting a new course for a system in crisis. Washington, DC: National Academies Press; 2013.

2. Neal RD. NICE prostate cancer clinical guideline: implications for primary care. Br J Gen Pract 2008; 58:607-8.

3. Klabunde CN, Ambs A, Keating NL, et al. The role of primary care physicians in cancer care. J Gen Intern Med 2009;24:1029-36.

4. Jang TL, Bekelman JE, Liu Y, et al. Physician visits prior to treatment for clinically localized prostate cancer. Arch Intern Med 2010;170:440-50.

5. Wallner LP, Abrahamse P, Uppal JK, et al. Involvement of primary care physicians in the decision making and care of patients with breast cancer. J Clin Oncol 2016:34:3969-75.

6. National Cancer Institute. Surveillance, Epidemiology, and End REsults Program. Cancer statistics factsheets: prostate cancer. Available from: http:// seer.cancer.gov/statfacts/html/prost.html. Accessed October 24, 2016.

7. Lin GA, Aaronson DS, Knight SJ, Carroll PR, Dudley RA. Patient decision aids for prostate cancer treatment: a systematic review of the literature. CA Cancer J Clin 2009;59:379-90.

8. Sommers BD, Beard CJ, D'Amico AV, Kaplan I, Richie JP, Zeckhauser RJ. Predictors of patient preferences and treatment choices for localized prostate cancer. Cancer. 2008;113:2058-67.

9. Hoffman RM, Shi Y, Freedland SJ, Keating NL, Walter LC. Treatment patterns for older veterans with localized prostate cancer. Cancer Epidemiol. 2015;39:769-77.

10. Floyd J, Fowler J, Collins MM, et al. Comparison of recommendations by urologists and radiation oncologists for treatment of clinically localized prostate cancer. JAMA 2000;283:3217-22.

11. Shahinian VB, Kuo Y-F, Freeman JL, Goodwin JS. Determinants of androgen deprivation therapy use for prostate cancer: role of the urologist. J Natl Cancer Inst 2006;98:839-45.

12. Kim SP, Gross CP, Nguyen PL, et al. Perceptions of active surveillance and treatment recommendations for low-risk prostate cancer: results from a national survey of radiation oncologists and urologists. Med Care 2014;52:579-85.

13. Cohen HJ. A model for the shared care of elderly patients with cancer. J Am Geriatr Soc 2009; 57(Suppl 2):S300-S2.

14. Sussman J, Baldwin LM. The interface of primary and oncology specialty care: from diagnosis through primary treatment. J Natl Cancer Inst Monogr 2010; 2010:18-24.

15. Smith GF, Toonen TR. Primary care of the patient with cancer. Am Fam Physician 2007;75:1207-14.

16. Bayliss EA, Ellis JL, Shoup JA, Zeng C, McQuillan DB, Steiner JF. Effect of continuity of care on hos- 
pital utilization for seniors with multiple medical conditions in an integrated health care system. Ann Fam Med 2015;13:123-9.

17. Nyweide DJ. Concordance between continuity of care reported by patients and measured from administrative data. Med Care Res Rev 2014;71:138-55.

18. Kruzikas D, Jiang HJ, Remus D, Barrett M, Coffey R, Andrews R. Preventable hospitalizations: a window into primary and preventive care, 2000. AHRQ publication no. 04-0056. Rockville, MD: Agency for Healthcare Research and Quality; 2004.

19. Donaldson M, Yordy K, Lohr K, Vanselow N. Primary care: America's health in a new era. Washington, DC: National Academies Press; 1996.

20. O’Toole E, Step MM, Engelhardt K, Lewis S, Rose $\mathrm{JH}$. The role of primary care physicians in advanced cancer care: perspectives of older patients and their oncologists. J Am Geriatr Soc 2009;57(Suppl 2): S265-S8.

21. Thompson I, Thrasher JB, Aus G, et al. Guideline for the management of clinically localized prostate cancer: 2007 update. J Urol 2007;177:2106-31.

22. Esnaola NF, Ford ME. Racial differences and disparities in cancer care and outcomes: where's the rub? Surg Oncol Clin N Am 2012;21:417-37, viii.

23. Saha S, Freeman M, Toure J, Tippens KM, Weeks C, Ibrahim S. Racial and ethnic disparities in the VA Health Care System: a systematic review. J Gen Intern Med 2008;23:654-71.

24. Zeliadt SB, Ramsey SD, Penson DF, et al. Why do men choose one treatment over another? A review of patient decision making for localized prostate cancer. Cancer 2006;106:1865-74.

25. Cruz M, Covinsky K, Widera EW, Stijacic-Cenzer I, Lee SJ. Predicting 10-year mortality for older adults. JAMA 2013;309:874-6.

26. Lu Ann Aday RA. A framework for the study of access to medical care. Health Serv Res 1974;9: 208-20.

27. Bach PB, Pham HH, Schrag D, Tate RC, Hargraves JL. Primary care physicians who treat blacks and whites. N Engl J Med 2004;351:575-84.

28. Freeman VL, Ricardo AC, Campbell RT, Barrett RE, Warnecke RB. Association of census tract-level socioeconomic status with disparities in prostate cancer-specific survival. Cancer Epidemiol Biomarkers Prev 2011;20:2150-9.

29. Royce TJ, Hendrix LH, Stokes WA, Allen IM, Chen RC. Cancer screening rates in individuals with different life expectancies. JAMA Intern Med 2014;174: $1558-65$.

30. American Cancer Society. Prostate cancer. Available from: https://old.cancer.org/acs/groups/cid/documents/ webcontent/003134-pdf.pdf. Accessed January 14, 2017.

31. Bill-Axelson A, Holmberg L, Filen F, et al. Radical prostatectomy versus watchful waiting in localized prostate cancer: the Scandinavian Prostate Cancer Group-4 randomized trial. J Natl Cancer Inst 2008; 100:1144-54.

32. Rosenbaum PR, Rubin DB. The central role of the propensity score in observational studies for causal effects. Biometrika 1983;70:41-55.

33. Mohler JL, Armstrong AJ, Bahnson RR, et al. Prostate cancer, version 1.2016. J Natl Compr Canc Netw 2016;14:19-30.

34. Klabunde CN, Han PKJ, Earle CC, et al. Physician roles in the cancer-related follow-up care of cancer survivors. Fam Med 2013;45:463-74.

35. Hickner J, Kent S, Naragon P, Hunt L. Physicians' and patients' views of cancer care by family physicians: a report from the American Academy of Family Physicians National Research Network. Fam Med 2007;39:126-31.

36. Ashton CM, Haidet P, Paterniti DA, et al. Racial and ethnic disparities in the use of health services. J Gen Intern Med 2003;18:146-52.

37. Nyarko E, Metz JM, Nguyen GT, Hampshire MK, Jacobs LA, Mao JJ. Cancer survivors' perspectives on delivery of survivorship care by primary care physicians: an internet-based survey. BMC Fam Pract 2015;16:143.

38. Mao JJ, Bowman MA, Stricker CT, et al. Delivery of survivorship care by primary care physicians: the perspective of breast cancer patients. J Clin Oncol. 2009;27:933-8.

39. Xu J, Neale AV, Dailey RK, Eggly S, Schwartz KL. Patient perspective on watchful waiting/active surveillance for localized prostate cancer. J Am Board Fam Med 2012;25:763-70.

40. de Bekker-Grob EW, Bliemer MCJ, Donkers B, et al. Patients' and urologists' preferences for prostate cancer treatment: a discrete choice experiment. Br J Cancer. 2013;109:633-40.

41. Aning JJ, Wassersug RJ, Goldenberg SL. Patient preference and the impact of decision-making aids on prostate cancer treatment choices and post-intervention regret. Curr Oncol 2012;19(Suppl 3):S37-44.

42. Anvik T, Holtedahl KA, Mikalsen H. "When patients have cancer, they stop seeing me"-the role of the general practitioner in early follow-up of patients with cancer-a qualitative study. BMC Fam Pract 2006;7:19.

43. Sada YH, Street RL, Singh H, Shada RE, Naik AD. Primary care and communication in shared cancer care: a qualitative study. Am J Manag Care 2011;17: 259-65. 
Appendix Table 1. Demographic and Clinical Characteristics of Responders and Non-Responders

\begin{tabular}{|c|c|c|c|}
\hline Characteristic & Responders N (\%) n = 2386 & Non-responders $\mathrm{N}(\%) \mathrm{n}=2286$ & $P$-value* \\
\hline Age (years) & & & 0.044 \\
\hline$<60$ & $704(29.5)$ & $712(31.2)$ & \\
\hline $60-64$ & $511(21.4)$ & $492(21.5)$ & \\
\hline $65-69$ & $559(23.4)$ & $464(20.3)$ & \\
\hline $70-74$ & $349(14.6)$ & $323(14.1)$ & \\
\hline$\geq 75$ & $263(11.0)$ & $294(12.9)$ & \\
\hline Missing & $0(0.0)$ & $1(0.0)$ & \\
\hline Race/ethnicity & & & $<0.0001$ \\
\hline Non-Hispanic White & $1850(77.5)$ & $1405(61.5)$ & \\
\hline Non-Hispanic Black & $391(16.4)$ & $691(30.2)$ & \\
\hline Hispanic & $32(1.3)$ & $88(3.9)$ & \\
\hline Non-Hispanic Other & $0(0.0)$ & $0(0.0)$ & \\
\hline Missing & $113(4.7)$ & $102(4.5)$ & \\
\hline Insurance & & & 0.058 \\
\hline Private & $1310(54.9)$ & $1198(50.9)$ & \\
\hline Medicaid & $73(3.1)$ & $102(4.5)$ & \\
\hline Medicare & $958(40.2)$ & $921(40.3)$ & \\
\hline None/Other & $19(0.8)$ & $20(0.9)$ & \\
\hline Missing & $26(1.1)$ & $45(2.0)$ & \\
\hline Gleason score & & & 0.317 \\
\hline$<7$ & $944(39.6)$ & $901(39.4)$ & \\
\hline 7 & $953(39.9)$ & $896(39.2)$ & \\
\hline$>7$ & $414(17.4)$ & $348(15.2)$ & \\
\hline Missing & $75(3.1)$ & $141(6.2)$ & \\
\hline Clinical tumor stage & & & 0.446 \\
\hline Stage 1 & $1706(71.5)$ & $1649(72.1)$ & \\
\hline Stage 2 & $574(24.1)$ & $514(22.5)$ & \\
\hline Stage 3 & $58(2.4)$ & $62(2.7)$ & \\
\hline Missing & $48(2.0)$ & $61(2.7)$ & \\
\hline \multicolumn{4}{|l|}{ Treatment } \\
\hline Surgery & $1230(51.6)$ & $1053(46.1)$ & 0.496 \\
\hline Missing & $386(16.2)$ & $543(23.8)$ & \\
\hline Radiation therapy & $703(29.5)$ & $602(26.3)$ & 0.539 \\
\hline Missing & $399(16.7)$ & $537(23.5)$ & \\
\hline Hormone therapy & $326(13.7)$ & $319(14.0)$ & 0.122 \\
\hline Missing & $423(17.7)$ & $566(24.8)$ & \\
\hline Active Treatment & & & $<0.001$ \\
\hline No & $393(16.5)$ & $567(24.8)$ & \\
\hline Yes & $1897(79.5)$ & $1620(70.9)$ & \\
\hline Missing & $96(4.0)$ & $99(4.3)$ & \\
\hline
\end{tabular}

*Using chi squared tests. 